\title{
Hybrid
}

Revue des arts et médiations humaines

$4 \mid 2017$

Malaise dans la représentation

\section{Châteaux andalous et Shoot in the Back: le cinéma de Lionel Soukaz comme représentation abjecte}

\section{Florian Grandena et Pascal Gagné}

\section{(2) OpenEdition}

1 Journals

\section{Édition électronique}

URL : https://journals.openedition.org/hybrid/888

DOI : $10.4000 /$ hybrid.888

ISSN : 2276-3538

Traduction(s) :

Châteaux andalous and Shoot in the Back: The films of Lionel Soukaz as abject representation - URL :

https://journals.openedition.org/hybrid/896 [en]

Éditeur

Presses universitaires de Vincennes

Référence électronique

Florian Grandena et Pascal Gagné, "Châteaux andalous et Shoot in the Back : le cinéma de Lionel Soukaz comme représentation abjecte », Hybrid [En ligne], 4 | 2017, mis en ligne le 04 septembre 2017, consulté le 13 avril 2022. URL : http://journals.openedition.org/hybrid/888 ; DOI : https://doi.org/ $10.4000 /$ hybrid. 888

Ce document a été généré automatiquement le 13 avril 2022.

Revue Hybrid 


\title{
Châteaux andalous et Shoot in the Back : le cinéma de Lionel Soukaz comme représentation abjecte
}

\author{
Florian Grandena et Pascal Gagné
}

«L'unique, c'est un geste radical, la souveraine
volée de bois vert, l'euphorie eucharistique des
expériences intérieures, la poésie qui défrise la
bonne société ${ }^{1}$."

1 Lorsqu'on demande à Lionel Soukaz pourquoi il provoque, il rétorque : «Quand tu t'en prends plein la gueule, tu réponds. [...] On nous interdisait de vivre donc on était prêt à tout pour pouvoir exister. Dans Ixe par exemple, si j'attaque le pape, ce n'est pas par hasard. Le pape condamnait l'homosexualité. Ensuite, il a condamné le préservatif, et on a vu les ravages en Afrique et dans d'autres pays catholiques. J'attaque des personnes qui ont un rôle politique que je trouve néfaste ${ }^{2}$. »

2 Aussi, la filmographie de ce réalisateur ne laisse généralement pas son public de marbre: son œuvre interpelle et suscite une réponse. Dans «Drague et amour. Sur www.webcam de Lionel Soukaz », Olivier Neveux relate un incident survenu après la projection du film éponyme devant un public d'universitaires et de conférenciers rassemblés en décembre 2009 à l'occasion d'un séminaire consacré à la transgression : quelques membres de ce parterre d'intellectuels manifestèrent un mécontentement et une grogne immédiats face à des images, somme toute relativement banales, d'internautes gai à la recherche d'une rencontre sexuelle, souvent nus, se masturbant parfois: "Cela fut cocasse: aucune réflexivité n'animait la fronde, comme si les ruptures et les transgressions ne devaient jamais être que théoriques ${ }^{3}$.» De toute évidence, le cinéma provenant de la marge n'est pas toujours facile à regarder - qu'il soit gai, pornographique ou expérimental.

3 On pourrait moquer cette attitude de "tolérance ", qui s'effarouche dès que le vulgaire est exhibé plutôt que conceptualisé. Bien que de telles réactions face au travail de Lionel Soukaz ne soient pas exceptionnelles (Olivier Neveux souligne un incident 
similaire survenu six ans auparavant après la projection d'un autre de ses films, Le Sexe des anges), on peut en effet s'interroger sur la ou les raison(s) du rejet d'œuvres qui, de notre point de vue, ne font rien d'autre que de mettre en scène la banalité et le ludique de la sexualité, de donner un temps et un espace momentanés à des corps gai parfois beaux, parfois imparfaits, d'âge et de taille différents, agissant comme objets et sujets de désir par l'entremise, par exemple, d'une webcam... Mais n'est-ce pas beaucoup déjà, pour des individus libéraux qui jugent l'homosexualité comme John Locke (1689) tolérait la prolifération de fausses religions, à l'exception de l'athéisme? C'est dire qu'on ne supporte la diversité que par l'acceptation forcée mais généreuse de travers et d'attitudes foncièrement blâmables, au prétexte que l'acte de censure inciterait à bien pire : la violence contre l'ordre social. Difficile, donc, de voir autre chose en cette tolérance qu'une stratégie supplémentaire de contrôle du corps de l'homosexuel docile, acceptable seulement s'il consent à rester confiné à la place littérale et symbolique assignée par la majorité. Un manque à combler provenant du fait que cette identité est constituée par la honte du stigmate ${ }^{4}$.

4 Le contrôle du corps homosexuel, avons-nous dit, semble de nos jours particulièrement apte à cristalliser les fantasmes paranoïaques, voire libidineux de plusieurs franges de la société, opposées ou complémentaires: fantasmes d'un corps musclé, épilé, déshabillé. Surface de projection du désir, le corps est constamment recadré. Car le physique du gai a son utilité : il est là, exposé sous toutes les coutures, pour flatter les libidos, adhérant ou contrevenant aux normes esthétiques de la santé et de la jeunesse, ou tout simplement pour amuser la galerie en faisant ce qui n'est pas attendu de lui. Il est ainsi, par sa réification même, discipliné, cantonné aux pages glacées d'un magazine ou à la scène bien délimitée d'un séminaire ou d'un plateau de télévision. Il est donc parfois difficile de voir dans cette nouvelle hypervisibilité ${ }^{5}$ du corps homosexuel autre chose qu'une forme supplémentaire d'assujettissement à un système visant la normalisation et la neutralisation d'une identité se définissant pourtant moins selon des politiques contestataires que par les effets du pouvoir.

5 Mais parfois, comme on peut l'observer dans le cinéma de Lionel Soukaz, l'individu cherche à se réapproprier le contrôle de soi par le biais de l'image et de l'autofilmage. Ne serait-ce pas quand l'homosexuel met en scène son corps, ses désirs, ses diverses manifestations selon ses propres termes, qu'il devient alors intolérable parce qu'il échappe à l'un des moyens de docilisation du groupe dominant, à savoir la bienséance, ciment de la pensée embourgeoisée? N'est-ce pas un peu contre ce désir de canalisation et de surveillance que cette œuvre du réalisateur se braque par le biais de tactiques transgressives? Il choisit en effet de filmer des scènes représentant des interdits et pratiques marginales, telles que la consommation d'héroïne ou les rapports homosexuels, car il conçoit le cinéma comme un exutoire au malaise social, une catharsis propre à l'acte de fuite : « Le cinéma est un média, un moyen d'aller vers les autres. C'est par lui que je me libère de mes grands secrets, c'est-à-dire de mon homosexualité6.»

6 Comme le suggère Michael Warner ${ }^{7}$, il faut se méfier de la normalisation du discours gai qui, dès le départ, s'était constitué en contre-public. Aujourd'hui, on le conçoit épanoui à proprement parler dans la sphère du domestique : une habitation privée, un endroit clos et discret à l'abri du regard d'autrui ${ }^{8}$. Renforçant ainsi une dichotomie traditionnelle mais déséquilibrée, la stratégie de la bienséance cherche à diriger l'homosexualité dans d'autres sites, réels ou virtuels, rendus inoffensifs. La 
représentation est en conséquence un moyen fréquemment utilisé pour domestiquer le privé en public, mais qui semble devenir foncièrement obscène lorsque le pubien est dévoilé. La construction idéologique d'une intimité somme toute privée est la projection répressive d'un imaginaire hétéronormatif dont il faut se méfier, car le sexe traverse d'ores et déjà la médiation d'un public dans le contact charnel. L'interdiction du sexe dans l'espace public trahit toujours un privilège'.

7 En pratiquant des tactiques de résistance dans ces endroits, réels ou virtuels, qui leur sont dévolus en partie par le groupe hétérocentriste majoritaire, les gais doivent, afin de se libérer et de s'émanciper ne serait-ce que temporairement, avoir recours à des tactiques précises, c'est-à-dire à « l'action calculée que détermine l'absence d'un propre [...]. La tactique n'a pour lieu que celui de l'autre. Aussi doit-elle jouer avec le terrain qui lui est imposé tel que l'organise la loi d'une force étrangère ${ }^{10}$ ». Pour ce qui a trait à l'œuvre analysée, de telles tactiques prennent la forme du filmage/auto-filmage constant, voire compulsif et obsessionnel du quotidien et de ce que ce dernier a de nonmontrable, de laid, de repoussant, mais aussi de ludique et de touchant ${ }^{11}$.

8 Ainsi, les films de Lionel Soukaz rendent abjects et le corps gai et le "propre » dans lequel celui-ci évolue, comme le suggère notre lecture des films Châteaux andalous et Shoot in the Back. Dans le premier, le réalisateur traverse le pays en bus avec son amant du moment, lequel se masturbe dans l'autocar; le caméraman également. Crescendo : on se rapproche en gros plan du pénis d'autrui. Il y a alternance entre les scènes d'attouchements et le paysage espagnol. Shoot in the Back est lui aussi filmé en séquence, mais sans interruption cette fois. Un anus qui défèque, fixé sur la pellicule, authentique, incessant. C'est de l'art brutal, sans recours à la manipulation du montage.

Les corps gai représentés ici sont perçus comme abjects dans le contexte des projections décrites en début de texte. Ils ne le sont pas de facto, c'est-à-dire sans qu'on ne prenne en compte les spectateurs et leur place au sein d'un lieu institutionnel comme l'université, ou d'un contexte de projection tel que la conférence. Il faut questionner les notions de tolérance mises à mal par des représentations abjectes - en tant qu'expressions libidinales et projet cinématographique. Ce double statut de la représentation comme abjection contamine le regard de ce public timoré, dégoûté par cette expérience d'une promiscuité presque complice ${ }^{12}$. 
fig. 1

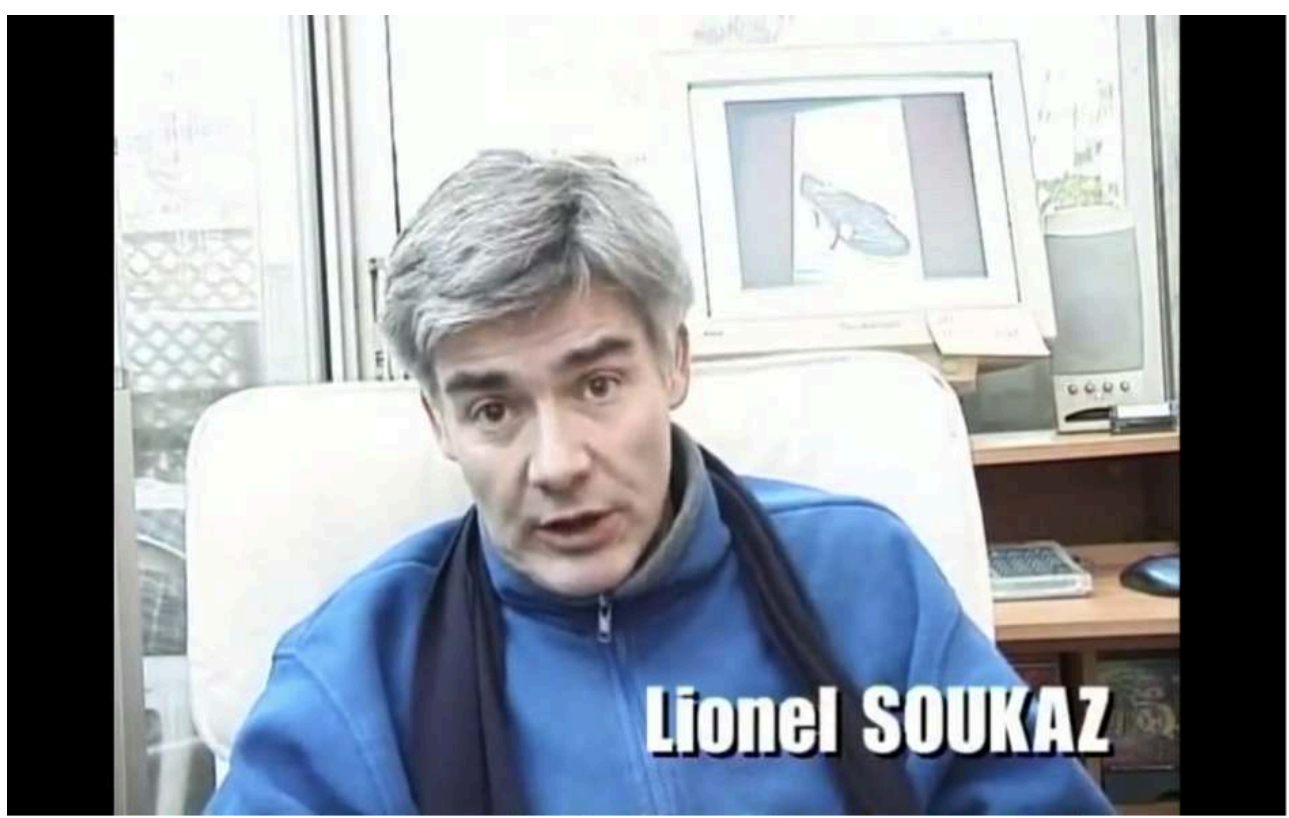

Lionel Soukaz sur YouTube.

(c) Lionel Soukaz

L'esthétique pornographique qui se développe à travers la répétition de gestes radicaux est une tactique cinématographique subversive : elle nous invite à repenser la notion de l'abject par la désublimation du corps, lorsque celui-ci est représenté de manière graphique. Le choix de ces deux films, retenus ici parmi un vaste corpus d'« immondices ", offre un contraste entre deux tabous en miroir, la verge et l'anus. En effet, la réception critique de ces films par son public pose le problème de la représentation qui tolère la différence mais ne l'accepte point. Cette réaction est typique d'une censure qui ne s'avoue pas comme telle, d'un confort dans le confinement du corps marginalisé à des stéréotypes, avec comme finalité le contrôle. Cette émancipation des clichés du corps gai - toujours beau, sain et propre, comique et ridicule - apparaît bien visible lors du moment de rupture que produit la caméra de Lionel Soukaz. Son regard ironique quant à la réification de l'identité homosexuelle s'affirme par la défiance de l'image cinématographique, quand il filme son propre corps avec une déférence accrue pour le détail, doté de surcroît d'un certain réalisme.

\section{Châteaux andalous : la désublimation d'une image graphique}

11 Il y a quelque chose d'utopique dans ce court film Châteaux andalous (5'47) et pas uniquement dans son titre, clin d'œil évident à l'expression "châteaux en Espagne » (mais aussi au film surréaliste de Luis Buñuel, Un chien andalou, 1929): le fantasme d'une homosexualité historiquement réprimée mais impossible à taire complètement, qui pourrait s'affranchir des normes de la bienséance comme celles des espaces utilisés normalement. Une orientation sexuelle dé-sublimée, en somme. Tandis que Freud (1905) concevait la sublimation comme un transfert des pulsions par la création et l'investissement d'objets socialement valorisés, la désublimation inverse ce rapport 
entre sexualité et morale; elle expose l'inadéquat dans le sexe, précisément pour choquer.

Châteaux andalous est divisé en quatre séquences entre lesquelles sont intercalés des écrans noirs d'environ 3-4 secondes, fonctionnant comme autant de moments de pause et de respiration. Si le film fait preuve d'une spontanéité indéniable (images tremblées, plans improvisés, action non feinte), le montage semble suggérer quant à lui la recherche d'un rythme au service de l'action filmée, les quatre séquences trahissant par leur durée croissante un intérêt proportionnel pour l'action principale.

13 Châteaux andalous tente de s'éloigner des normes de contrôle des corps au sein d'espaces précis en filmant des fragments de ces corps saisis dans leur action subversive. D'ailleurs, plusieurs courts métrages de Soukaz consistent en fait en des segments censurés de ses longs métrages. Le film débute par un générique de quatre secondes, fait d'images tremblées d'une feuille de cahier d'école sur laquelle sont griffonnés au stylo le titre et le nom du cinéaste. Commence ensuite la première séquence de 26 secondes qui permet au réalisateur de poser son décor : un autocar traversant la campagne andalouse embrumée. Il se représente alors avec son compagnon comme les protagonistes d'un film de vacances. S'ensuit une scène de masturbation dans le transport, soit la subversion d'un non-lieu par un acte homosexuel ${ }^{13}$. Les plans larges de la campagne espagnole composant cette première séquence sont brièvement interrompus à la $26^{\mathrm{e}}$ seconde par un gros plan des mains des deux figurants sur l'entrejambe du partenaire de Lionel Soukaz. La caméra s'intéresse au visage de l'un des hommes regardant par la fenêtre de l'autocar défiler les collines.

14 La deuxième séquence (50") commence par un plan de profil du compagnon de route. Alors que le véhicule vient de s'immobiliser au centre d'une petite bourgade, la caméra scrute son environnement immédiat, un square, une façade d'église, et glisse rapidement vers un groupe de jeunes hommes pour enfin s'arrêter sur le fessier de l'un d'eux, rebondi et moulé dans un jean. C'est aussi avec des plans furtifs de la campagne andalouse que débute la troisième séquence (1'36). Alors que l'autobus reprend la route, la lentille nous offre un plan rapproché du sexe tendu, sous un sous-vêtement noir. La caméra dans la main droite, Lionel Soukaz s'affaire avec son autre main à dégager et masturber la verge durcie, avant que son amant ne reprenne la besogne. La dernière séquence (2'35) est constituée d'un plan rapproché de la caresse, filmé sans coupure. La caméra passe du visage de l'homme à son sexe, l'attouchement atteignant rapidement un paroxysme explosif et jubilatoire couronné par un gros plan aussi bref que synchrone, pour retourner ensuite, non sans humour, vers le paysage andalou. 
fig. 2

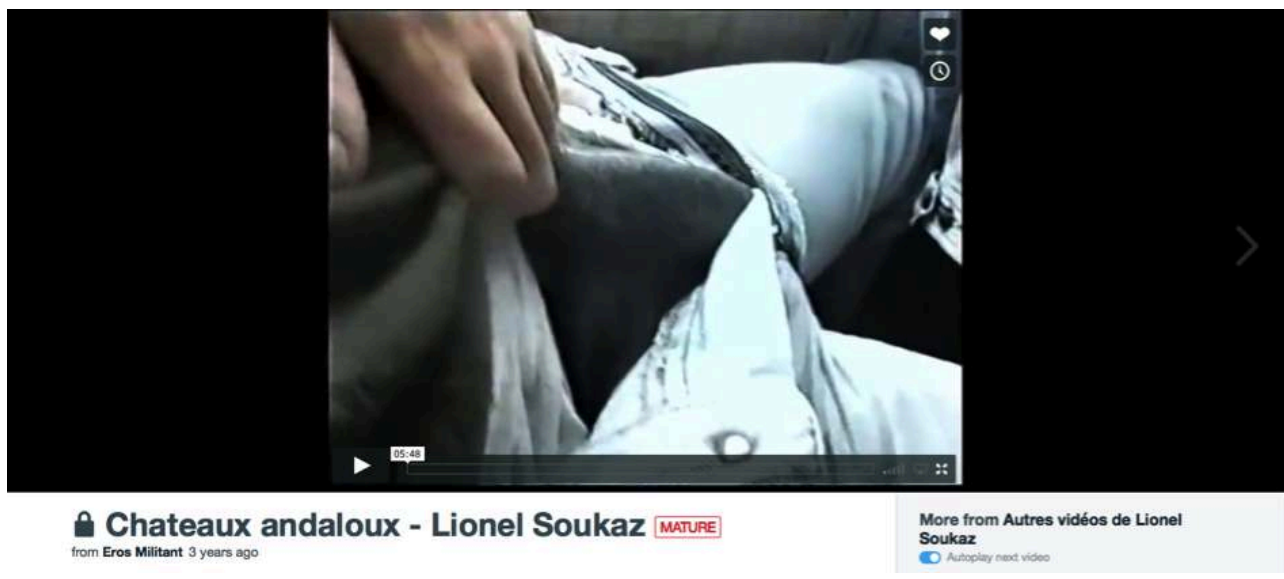

(c) Lionel Soukaz symbole traditionnel d'instrument de pouvoir et de domination, que Lionel Soukaz dépeint avec un ludisme puéril, et la main, outil de manœuvre, moyen de création et de soulagement. Ce sont à la fois le poing et le pénis en tant qu'organes sexuels qui se trouvent dé-sublimés en un non-lieu " inapproprié », rendu abject par l'action filmée ${ }^{14}$ Châteaux andalous brouille donc les codes de conduite à plusieurs égards. avec l'hypervisibilité gai du tournant du siècle. Cela devient plus évident encore lorsqu'on revisite les conceptualisations victoriennes de l'homosexualité comme inversion pathologique du féminin ${ }^{15}$.

Sur la pellicule, la désublimation de l'organe se fait par le travail littéral de la paume qui, laborieusement, se transforme ici en vecteur de transgression: de la même façon que Lawrence R. Schehr a parlé des ressemblances entre la main de l'écrivain et celle de l'homme se masturbant ${ }^{16}$, nous voudrions comparer l'activité du voyeur taciturne et celle de son acolyte se caressant : deux occupations spontanées et solitaires, librement choisies et exercées, s'appuyant sur la mécanique des doigts et sur l'extension d'un pénis ou d'un objectif de caméra. Ce rapprochement "ontologique» explique la vulgarité de la représentation: elle rend profane ce qu'il y a de plus sacré dans le transport en commun.

\section{Shoot in the Back : approcher et filmer l'indigeste} sexuel soi-disant inapproprié, Shoot in the Back met l'accent sur la monstration transgressive d'une action somme toute banale en un lieu jugé acceptable. Un autre interdit est enfreint : ce film dépeint non plus la verge roide, mais l'anus entrouvert. Comble du vulgaire, de l'obscène, la caméra expose un acte pudique, quotidien, fonctionnel et objectal. C'est un cinéma de l'affect qui met en avant le fétiche comme signe de l'image-pulsion ${ }^{17}$.

Le fétiche, en réalité, explique l'assemblage par les processus de réification et de réduction, par la substitution et le désir d'une relation impossible. L'homme-trou-ducul. Shoot in the Back est peut-être la production de Soukaz manquant le plus

Hybrid, 4 | 2017 
d'humanité, puisque le regard est privé de visage et de langage. Ce court métrage de quelques minutes nous montre en très gros plan un anus se dilatant face à un objectif scrutateur et impitoyable, sans interruption ou compromis possible - puisque cet anus et l'action de ce dernier, filmés en un plan séquence unique, occupent à eux seuls tout l'espace et le temps écraniques. Le cadrage focal est entièrement consacré à l'ouverture lente et inéluctable de l'orifice, ces lèvres desquelles on voit poindre la matière fécale. Comme une conversation impossible.

Shoot in the Back n'est certainement pas la première œuvre qui, par la monstration et l'utilisation de résidus organiques, cherche à déstabiliser les normes du montrable et du non-montrable, de l'intérieur et de l'extérieur (du corps en l'occurrence), du bon et du mauvais goût. Il s'agit d'une double attaque du sujet et de l'écran pour retourner à une vérité de l'abject en dehors du littéral ${ }^{18}$. Pensons par exemple aux célèbres Piss Paints d'Andy Warhol (1977-1978), au Piss Christ (1987) d'Andres Serrano, ainsi qu'aux sculptures de sang congelé de Mark Quin (Self, 1991, 1996, 2001) et aux vomissements performatifs plus récents de Millie Brown. Au cinéma, il suffit de mentionner Le Satyricon de Federico Fellini (1979) et Salò ou les 120 journées de Sodome de Pier Paolo Pasolini (1975). Dans le premier, on voit un citoyen de la Rome antique déféquer dans la rue, dans le second, l'élite se gaver d'excréments; l'un et l'autre critiquent la bourgeoisie et le fascisme (qui plus est, dans un contexte où l'homosexualité réprimée est au centre de l'imaginaire collectif). Soulignons aussi que l'activisme gai des années 1980 et 1990 faisait régulièrement usage de l'obscène et des fluides corporels pour déstabiliser le politique ${ }^{19}$.

21 Si l'œuvre scatologique revêt une dimension dérangeante ou subversive, c'est moins à cause de l'action (somme toute insignifiante en cet endroit) que du filmage de l'acte, attaquant ainsi de front la bienséance inhérente à certaines fonctions corporelles et à certains de leurs lieux associés. Il n'y a pas de mise en garde : l'activité qui s'y déroule, discrète et honteuse dans sa quotidienneté, prend une dimension abjecte à cause de la tactique de subversion de la pellicule. Le critique pardonne au final plus facilement l'abjection rétrospective de Fellini ou de Pasolini que celle de Soukaz, pourtant tout aussi absurde.

Les normes auxquelles s'attaque Shoot in the Back relèvent du partage de l'espace au sein duquel le corps expie dans la raison occidentale : les cabinets ${ }^{20}$. Alors qu'il devrait être dédié au soulagement discret et privé des individus, ce non-lieu est déstabilisé par le regard impudique de la caméra, la capture de ce qui devrait rester non montrable ${ }^{21}$. En effet, si le périmètre restreint du site de tournage n'est pas à proprement parler rendu transgressif par l'activité du corps qui excrète là où il se doit, c'est bien par la documentation que cette expulsion s'affiche comme subversive. Ainsi, d'un lieu à la fois intime et public où règnent habituellement la discrétion et la honte, le petit espace du périnée devient, par le biais de l'enregistrement de la caméra, la scène minimaliste et dramatique où est glorifiée la souillure.

Si l'anus est sans nul doute le protagoniste de ce court métrage tout aussi indiscret que transgressif, il n'en est pas moins désincarné. Réduit à sa fonction primaire d'éjection de déchets fécaux, l'organe devient métonymie du corps de l'homme gai filmé et se filmant, car l'auteur du court métrage en est à la fois le sujet et l'objet. Encore une fois : de cet homme on ne voit nullement les traits, le visage reste hors champ, et on n'en entend aucun mot, les seuls sons audibles étant ceux, scatologiques, émis régulièrement par l'organe. Sans visage, mais aussi sans langage, le protagoniste 
demeure non identifiable, n'existant que par son cul et rendant de ce fait l'expérience de la défécation aussi déshumanisée qu'universelle. En gros plan, ce fétiche est exemplaire du grossier qui nous affecte et nous révulse dans l'imaginaire social, en plus de renvoyer le critique de cinéma à la violence symbolique du goût ${ }^{22}$.

Cependant, cet anus et sa place littérale et métaphorique à l'écran font de Shoot in the Back un film proche de l'impropre archaïque et élémentaire ${ }^{23}$. Le déchet organique et son éjection nous dérangent et nous captivent tout à la fois. Leur cadrage, leur examen sans pause et sans compromis nous sont intolérables, car nous voyons comment le corps d'autrui, et donc le nôtre, peuvent être réduits à leur état le plus primitif. Un peu comme la présence d'un cadavre, la merde filmée correspond à « la mort infestant la vie » et représente «le danger venu de l'extérieur de l'identité : le moi menacé par du non-moi, la société menacée par son dehors, la vie par la mort $»^{24}$. En tant que sujets, nous ne pouvons qu'être dérangés par cet organe aux fonctions abjectes ${ }^{25}$ dont la suite continue d'images nous force à regarder ce que nous ne pouvons ou ne désirons pas reconnaitre ${ }^{26}$.

Si la société valorise parfois à l'extrême le phallus (sa possession, sa réappropriation et le désir de le conserver), elle aspire en même temps à sublimer et à garder dans la sphère privée l'anus, la zone cachée, honteuse, secrète du bourgeois. En tant qu'organe dissimulé par excellence, l'anus est sublimé, lieu du désir honteux; et ce désir, dans la société bourgeoise, est contrôlé par le biais de sa sublimation. Aussi, l'anus libéré serait capable de déstabiliser l'ordre social phallocratique, car «notre trou du cul n'est pas honteux ni personnel, il est public et révolutionnaire ${ }^{27} »$. L'une des zones érogènes masculines revendiquées comme telles par l'homosexuel, ce défet du joug idéologique peut évincer tout cadre moralisateur.

Les images de Soukaz témoignent d'un refus d'enfermement du sujet queer dans des représentations tolérantes de l'homosexualité, devenue propre, dépolitisée, inoffensive : aux antipodes des mécanismes mercantiles d'entraînement à la docilité ou de la critique de droite, le corps, chez Soukaz, se libère en produisant non pas des étiquettes ou de la main-d'œuvre, mais de l'abjection et du gaspillage. Le caractère abject du sperme et de l'excrément, de même que la transgression des normes relatives au partage du sensible, résulte de la performance d'un nouveau sens esthétique. L'abjection est filmée de manière réaliste, en gros plan, en plan séquence, l'absence de médiation renforçant l'illusion d'une authenticité de l'image et de sa véracité. Le fétiche dans la représentation passe encore pour une critique révolutionnaire du spectacle s'il s'oppose à la marchandisation de l'existence en tant que simulation, du moins tel que le préconisait Guy Debord ${ }^{28}$.

\section{Un cinéma capricieux}

Châteaux andalous et Shoot in the Back s'opposent et se répondent: tandis que Châteaux andalous est marqué par son mouvement fluide dans l'arrière-pays bucolique, Shoot in the Back est caractérisé par une immobilité du corps (seule la caméra bouge) au sein de l'espace clos des toilettes. Dans les deux films, aucune énonciation; tout juste le souffle diégétique, auquel se mêlent plaisir et soulagement physiques poussés à leur paroxysme de l'homme jouissant ou déféquant. La phrase est absente, le mot même est ici absent, laissant place à ces images d'exaltation. Le corps homosexuel semble s'émanciper donc aussi du joug du langage qui catégorise, et de la réduction de ses 
formes d'existence par l'étroitesse d'un rapport à la politesse ou à l'exotique. Comme le suggère David $\mathrm{M}$. Halperin ${ }^{29}$, une telle abjection met la capacité d'endurance à l'épreuve pour mieux résister à l'humiliation sociale de la différence.

Mais une telle tactique d'émancipation et de libération du corps gai n'est-elle pas vaine? Nous savons que la tactique, par sa nature spontanée et temporaire, opère par les interstices et les moments de répit: «Elle fait du coup par coup. Elle profite des "occasions" et en dépend, sans base où stocker des bénéfices, augmenter un propre et prévoir des sorties. Ce qu'elle gagne ne se garde pas $^{30}$. " Ne pouvant conserver ce qu'elle conquiert, cette tactique serait vouée à un échec relatif si elle n'était rendue à la postérité par le biais du filmage. Dans les faits, la caméra permet à la fois de fixer pour l'éternité les objets et les sujets se trouvant devant son objectif et de regarder ou de projeter ces images à l'envi, comme fantasme d'une intériorité enfin maniable. On entre ainsi en dialogue avec l'autre par l'affirmation de soi-même. C'est bien la prétention de l'enfant terrible : «Mon cinéma raconte ma vie, mais aussi la manière de faire sa révolution sur soi-même et de la faire partager par les autres ${ }^{31}$.» Le corps comme révolution, c'est donc le renversement de l'esthétique bourgeoise au profit d'une vérité alternative, abjecte et quelque peu traumatique.

La revendication d'une représentation abjecte, dans l'esprit de provoquer l'embourgeoisement d'un public qui se veut pourtant tolérant, s'efforce vainement de subvertir le regard. Serait-ce donc la nature irrémédiable et imparable d'une telle tentative d'émancipation ainsi déployée qui a pu choquer le groupe d'universitaires mentionné par Olivier Neveux (en d'autres termes, serait-ce la reconnaissance de la réussite même de la tactique qui l'aurait horripilé) ? Seraient-ce les images crues et laides, spontanées et tremblées, qui remueraient viscéralement la sensibilité naïve et le bon goût? Ou serait-ce encore la vitalité insolente de l'artiste et sa franchise impertinente? «Ce qui ne pardonne pas dans les films de Soukaz est bien leur absence de toute sentimentalité, la mise au jour d'un autre imaginaire, moins mensonger, car à la hauteur de la somme d'artifices, de montages, d'apparences, de mises en scène, de duperies élégantes, de feintes et de jeu qui composent la vie érotique $^{32}$.» Tactique réussie, donc dérangeante, des images brutes ou de l'absence totale de délicatesse : un cinéma appauvri qui s'impose, excédant nos limites, notre tolérance. Il problématise la représentation pudique du corps gai, à la fois marginale et dépolitisée, comme esthétique révolutionnaire ${ }^{33}$.

\section{BIBLIOGRAPHIE}

AUGÉ Marc, Non-lieux, introduction à une anthropologie de la surmodernité, Paris, Seuil, 1992.

BERLANT Lauren et WARNER Michael, «Sex in Public », Critical Inquiry, vol. 24, n 2, 1998,

p. 547-566.

BORDOWITZ Gregg, The AIDS Crisis is Ridiculous : And Other Writings, 1986-2003, Cambridge, The Massachussetts Institute of Technology Press, 2004. 
BoURDIEU Pierre, La Domination masculine, Paris, Seuil, 1998.

CERTEAU (de) Michel, L’Invention du quotidien. I. Arts de faire, Paris, Gallimard, 1990.

DEBORD Guy, La Société du spectacle, Paris, Buchet-Chastel, 1967.

DELEUZE Gilles, L'Image-mouvement. Cinéma 1, Paris, Minuit, 1983.

FOSTER Hall, « Obscene, Abject, Traumatic », October, vol. 78, 1996, p. 106-124.

FREUD Sigmund, Trois essais sur la théorie sexuelle [1905], Paris, Flammarion, 2011.

GAFFEZ Fabien, « Un chant d'amour : la morale du voleur », Positif, vol. 645, 2004, p. 90-97.

HALLAS Roger, « AIDS and Gay Cinephilia », Camera Obscura, vol. 18, n 52.1, 2003, p. 84-127.

HALPERIN David M., What Do Gay Men Want? An Essay on Sex, Risk, and Subjectivity, Ann Arbor, University of Michigan Press, 2007.

HAWHEE Debra, Moving Bodies : Kenneth Burke at the Edges of Language, Columbia, University of South Carolina Press, 2009.

HOCQUENGHEM Guy, La Dérive homosexuelle, Paris, Jean-Pierre Delarge, 1977.

HOCQUENGHEM Guy, Le Désir homosexuel, Paris, Fayard, 2000.

HUMPHREYS Laud, Tearoom Trade : A Study of Homosexual Encounters in Public Places, New Brunswick (É.-U.)/Londres, Transaction Publisher, 1975.

KRISTEVA Julia, Pouvoirs de l'horreur, Paris, Seuil, 1980.

LOCKE John, Letter Concerning Toleration [1689], Amherst, Prometheus Books, 1990.

LONG Thomas L., "Plague of Pariahs : AIDS'zines and the Rhetoric of Transgression », Journal of Communication Inquiry, vol. 24, $\mathrm{n}^{\circ} 4,2000$, p. 401-411.

MELINARD Michaël, «Lionel Soukaz fait son souk à Pantin », L'Humanité, 12 juin 2004. [En ligne] http://www.humanite.fr/node/307211 [consulté le 22 mai 2017].

NEVEUX Olivier, « Drague et amour. Sur www.webcam de Lionel Soukaz », in Jean-Marc LACHAUD et Olivier NEVEUX (dir.), Une esthétique de l'outrage ?, Paris, L'Harmattan, 2012, p. 91-105.

PIDDUCK Julianne, «The Visible and the Sayable : The Moment and Conditions of Hypervisibility ", in Florian GRANDENA et Christine JOHNSTON (dir.), Cinematic Queerness : Gay and Lesbian Hypervisibility in Contemporary Francophone Feature films, Oxford, Peter Lang, 2011, p. 9-40.

sCHEHR Lawrence R., Parts of an Andrology : On Representations of Men's Bodies, Stanford, Stanford University Press, 1997.

WARNER Michael, The Trouble with Normal : Sex, Politics, and the Ethics of Queer Life, Cambridge, Harvard University Press, 2000.

WARNER Michael, Publics and Counterpublics, Cambridge, Zone, 2005.

WATNEY Simon, Practices of Freedom : Selected Writings on HIV/AIDS, Durham, Duke University Press, 1994.

WATNEY Simon, Policing Desire : Pornography, AIDS, and the Media, Londres, Continuum International Publishing Group, 1997. 


\section{ANNEXES}

\section{Filmographie}

Luis Buñuel, Un chien andalou, 1929.

Federico Fellini, Le Satyricon, 1969.

Pier Paolo Pasolini, Salò, ou les 120 jours de Sodome, 1975.

Lionel Soukaz, Le Sexe des anges, 1977.

Lionel Soukaz, Châteaux andalous, 1995.

Lionel Soukaz, www.webcam, 2005.

Lionel Soukaz, Shoot in the back, 2006.

\section{NOTES}

1. Fabien Gaffez, « Un chant d'amour : la morale du voleur », Positif, $n^{\circ}$ 645, 2014, p. 97.

2. Michaël Melinard, «Lionel Soukaz fait son souk à Pantin », L'Humanité, 12 juin 2004. [En ligne] http://www.humanite.fr/node/307211 [consulté le 22 mai 2017].

3. Olivier Neveux, «Drague et amour. Sur www.webcam de Lionel Soukaz ", in Jean-Marc Lachaud et Olivier Neveux (dir.), Une esthétique de l'outrage ?, Paris, L'Harmattan, 2012, p. 91.

4. " Gay subjectivity is divided against itself, formed in stigma, in rejection by others - especially by those whom one desires - and by oneself. Our very loves and pleasures are constituted in relation to part ourselves that are causes of irredeemable shame in our social experience of them » (David M. Halperin, What Do Gay Men Want? An Essay on Sex, Risk, and Subjectivity, Ann Arbor, University of Michigan Press, 2007, p. 69).

5. Julianne Pidduck, "The Visible and the Sayable: The Moment and Conditions of Hypervisibility ", in Florian Grandena et Christine Johnston (dir.), Cinematic Queerness : Gay and Lesbian Hypervisibility in Contemporary Francophone Feature films, Oxford, Peter Lang, 2011, p. 9-40.

6. Michaël Melinard, «Lionel Soukaz fait son souk à Pantin », L'Humanité, 12 juin 2004. [En ligne] http://www.humanite.fr/node/307211 [consulté le 22 mai 2017]. Sur les rapports symboliques entre l'expression corporelle abjecte d'une part, et l'acte cathartique de la création artistique d'autre part, on renvoie à l'excellent travail de Debra Hawhee sur l'œuvre littéraire de Kenneth Burke : Debra Hawee, Moving Bodies: Kenneth Burke at the Edges of Language, Columbia, University of South Carolina Press, 2009.

7. Michael Warner, The Trouble with Normal: Sex, Politics, and the Ethics of Queer Life, Cambridge, Harvard University Press, 2000 ; Id., Publics and Counterpublics, Cambridge, Zone, 2005.

8. Vouloir imposer la bienséance, c'est satisfaire un système normatif visant à gérer et à contrôler les comportements et les corps des individus pour le bon fonctionnement de la société ou, en d'autres termes, une stratégie du groupe dominant (hétérosexuel) que Michel de Certeau définit de la façon suivante: «Le calcul (ou la manipulation) des rapports de force qui devient possible à partir du moment où un sujet de vouloir et de pouvoir (une entreprise, une armée, une cité, une institution politique) est isolable. Elle postule un lieu susceptible d'être circonscrit comme un propre et d'être la base d'où gérer les relations avec une extériorité de cibles ou de menaces (les clients ou les concurrents, les ennemis, la campagne autour de la ville, les objectifs et objets de recherche) " (Michel de Certeau, L'Invention du quotidien, I. Arts de faire, Paris, Gallimard, 1990, p. 85).

9. Lauren Berlant et Michael Warner, "Sex in Public ", Critical Inquiry, vol. 24, n², 1998, p. 547-566. 
10. Michel de Certeau, L'Invention du quotidien, I. Arts de faire, Paris, Gallimard, 1990, p. 86.

11. Ces deux courts sont représentatifs de l'esthétique «bricolage » revendiquée dans l'œuvre de Soukaz de ces dernières années, ainsi que d'une économie de moyens (rendue possible grâce à l'utilisation de la technologie numérique) et d'une liberté de création absolue.

12. «Ce n'est donc pas l'absence de propreté ou de santé qui rend abject, mais ce qui perturbe une identité, un système, un ordre. Ce qui ne respecte pas les limites, les places, les règles. L'entre-deux, l'ambigu, le mixte » (Julia Kristeva, Pouvoirs de l'horreur, Paris, Seuil, 1980, p. 12).

13. Les non-lieux sont des espaces interchangeables dans lesquels évoluent des individus anonymes. Ce sont des lieux de transit par excellence, à savoir aussi bien l'infrastructure nécessaire à la circulation accélérée des individus et des biens (voies rapides, échangeurs, aéroports) que les moyens de transport eux-mêmes, les grands centres commerciaux et même les camps de réfugiés (Marc Augé, Non-lieux, introduction à une anthropologie de la surmodernité, Paris, Seuil, 1992, p. 48).

14. Guy Hocquenghem (Le Désir homosexuel, Paris, Fayard, 2000) explique que le capitalisme, pour pouvoir survivre, doit canaliser les relations entre individus en faisant appel au complexe d'œEdipe qui a pour objectif de structurer et de contrôler le désir. La société et les individus la composant sont ainsi œdipianisés et s'appuient sur l'attribution de certaines fonctions à certains organes : remplissant le même rôle que l'argent au sein du capitalisme, le phallus devient un organe social et impose un mode relationnel basé sur la hiérarchie entre individus (ibid., p. 95). Ainsi sublimés, les désirs sexuels et certains organes (ici, la main et le pénis de l'homosexuel) sont repoussés dans la sphère privée et toute tentative de les en faire sortir ne peut être vue que comme une tentative de transgression des normes de la bienséance.

15. «Les homosexuels savent qu'ils jouissent, hors du système... Ils savent et ils formulent très vite qu'ils sont des traîtres, au regard d'une société normale : ils ont les caractères physiques par lesquels l'homme prétend montrer sa supériorité (le pénis) et ils sont méprisés, comme les femmes auxquelles ils savent qu'ils seront assimilés par les hommes "virils" " (Guy Hocquenghem, La Dérive homosexuelle, Paris, Jean-Pierre Delarge, 1977, p. 41).

16. Lawrence R. Schehr, Parts of an Andrology : on Representations of Men's Bodies, Stanford, Stanford University Press, 1997, p. 109-147.

17. Gilles Deleuze, L'Image-mouvement, Cinéma 1, Paris, Minuit, 1983.

18. Hall Foster, «Obscene, Abject, Traumatic », October, vol. 78, 1996, p. 106-124.

19. Voir par exemple Gregg Bordowitz, The AIDS Crisis is Ridiculous : And Other Writings, 1986-2003, Cambridge, The Massachussetts Institute of Technology Press, 2004 ; Thomas L. Long, " Plague of Pariahs: AIDS'zines and the Rhetoric of Transgression ", Journal of Communication Inquiry, vol. 24, $\mathrm{n}^{\circ} 4$, 2000, 401-411 ; Simon Watney, Practices of Freedom : Selected Writings on HIV/AIDS, Durham, Duke University Press, 1994 ; et Simon Watney, Policing Desire: Pornography, AIDS, and the Media, Londres, Continuum International Publishing Group, 1997.

20. « Un coin d'irrationnel » (Michel de Certeau, L'Invention du quotidien. I. Arts de faire, Paris, Gallimard, 1990), un espace ambigu, puisque fondamentalement intime, honteux et public en même temps. Mais aussi, un endroit excitant et dangereux. Voir également l'étude classique de Laud Humphrey sur les rapports sexuels anonymes d'hommes gai dans des toilettes publiques (Laud Humphreys, Tearoom Trade: A Study of Homosexual Encounters in Public Places, New Brunswick (É.-U.)/Londres, Transaction Publisher, 1975.

21. Nous considérons ici les toilettes publiques comme des non-lieux puisque leur raison d'être et leur utilisation ne sont pas identitaires (en tout cas pas systématiquement car les toilettes peuvent être des espaces genrés), ni relationnels, ni historiques (Marc Augé, Non-lieux, introduction à une anthropologie de la surmodernité, Paris, Seuil, 1992, p. 100).

22. Pierre Bourdieu, La Domination masculine, Paris, Seuil, 1998.

23. « Dégoût d'une nourriture, d'une saleté, d'un déchet, d'une ordure. Spasmes et vomissements qui me protègent. Répulsion, haut-le-cœur qui m'écarte et me détourne de la souillure, du 
cloaque, de l'immonde. Ignominie de la compromission, de l'entre-deux, de la traîtrise. Sursaut fasciné qui m’y conduit et m'en sépare» (Julia Kristeva, Pouvoirs de l'horreur, Paris, Seuil, 1980, p. 10).

24. Julia Kristeva, Pouvoirs de l'horreur, Paris, Seuil, 1980, p. 12 et 86.

25. Les fonctions anales sont abjectes, dans le sens où elles bouleversent les normes du montrable et du bienséant en révélant l'intérieur à l'extérieur et en réduisant l'écart entre le vivant (le corps) et l'inerte/le mort (l'excrément).

26. «S'il est vrai que l'abject sollicite et pulvérise tout à la fois le sujet, on comprend qu'il éprouve dans sa force maximale lorsque, las de ses vaines tentatives de se reconnaitre hors de soi, le sujet trouve l'impossible en lui-même : lorsqu'il trouve que l'impossible, c'est son être même, découvrant qu'il n'est autre qu'abject » (Julia Kristeva, Pouvoirs de l'horreur, Paris, Seuil, 1980, p. 12).

27. Guy Hocquenghem, La Dérive homosexuelle, Paris, Jean-Pierre Delarge, 1977, p. 44.

28. Guy Debord, La Société du spectacle, Paris, Buchet-Chastel, 1967.

29. "If... abjection names the social situation that forces us, in order to survive, to resist the crushing burden of shame, to glory in our exclusion from the scene of social belonging, to transcend (at least in our imagination) the humiliating realities of social existence, and to find in the secret history of our pleasures a source of personal and collective triumph over the forces that would destroy us, then abjection would seem to have some life-enhancing uses " (David M. Halperin, What Do Gay Men Want? An Essay on Sex, Risk, and Subjectivity, Ann Arbor, University of Michigan Press, 2007).

30. Michel de Certeau. L'Invention du quotidien. I. Arts de faire, Paris, Gallimard, 1990, p. 86-87.

31. Michaël Melinard, «Lionel Soukaz fait son souk à Pantin », L'Humanité, 12 juin 2004. [En ligne] http://www.humanite.fr/node/307211 [consulté le 22 mai 2017].

32. Olivier Neveux, « Drague et amour. Sur www.webcam de Lionel Soukaz », in Jean-Marc Lachaud et Olivier Neveux (dir.), Une esthétique de l'outrage ?, Paris, L'Harmattan, 2012, p. 102.

33. «The gay cinephilia [...] emerges precisely as a recovery of history, a means to articulate contemporary gay structures of feeling in the AIDS epidemic through the visual archive that has played a significant role in the constitution and maintenance of postwar gay identities and subcultures » (Roger Hallas, "AIDS and Gay Cinephilia », Camera Obscura, vol. 18, n 52.1, 2003, p. 118).

\section{RÉSUMÉS}

L'œuvre iconoclaste du cinéaste Lionel Soukaz embrasse du regard toutes les expressions abjectes du corps homosexuel telles que l'éjaculation ou l'excrétion. Son cinéma, outrageant, remet en perspective le contexte contemporain de la pseudo-tolérance, surtout quand on constate que l'on s'effarouche encore de l'exposition d'organes mis à nu par la caméra. Par le jeu de la représentation comme exutoire et performance, l'artiste tente donc d'extérioriser et de penser la sexualité gai comme forme de vie et comme pratique révolutionnaire. En effet, ses films focalisent sur la transgression des normes public-privé, de même que celles relatives aux normes de la bienséance dans la mise en scène de la représentation. Son processus créatif : le cinéma comme une sortie de soi, pour entrer en communication avec l'autre, en tant que geste révolutionnaire. Il en va du statut ontologique de la pellicule comme forme purement abjecte. 
INDEX

Mots-clés : abjection, cinéma, homosexualité, Lionel Soukaz, théorie queer

\section{AUTEURS}

\section{FLORIAN GRANDENA}

Florian Grandena est professeur associé au département de communication de l'Université d'Ottawa (Canada). Sa recherche actuelle porte sur les représentations des homosexualités dans le cinéma français contemporain, principalement les fictions d'olivier Ducastel et Jacques Martineau, sur lesquels il prépare un livre, et les productions de Lionel Soukaz. Il s'intéresse aussi à la figure du vampire dans le cinéma international et aux productions visuelles de musiciennes pop telles qu'Annie Lennox. Il est par ailleurs l'initiateur du cycle de conférences « Hypervisibilité » qui se consacre à la représentation des homosexualités dans les cultures visuelles transnationales. Il est, avec Cristina Johnston (University of Stirling, GB), le coéditeur de New Queer Images: Representations of homosexualities in Contemporary Francophone Visual Cultures et Cinematic Queerness: Gay and lesbian Hypervisibility in Contemporary Francophone Feature Films (Peter Lang, 2011) et avec Charles Batson (Union College, GB), le coéditeur d'un numéro spécial de Contemporary French Civilization (vol. 37.2-3, 2012) dédié aux travaux et à la mémoire de Lawrence R. Schehr. Il a aussi été titulaire d'une chaire internationale au Labex Arts-H2H en 2014, et il est directeur du groupe de recherche ÆPI (esthétique et et politique de l'image (http://aepiuottawa.weebly.com).

\section{PASCAL GAGNÉ}

Pascal Gagné poursuit ses études doctorales au département de communication de l'Université d'Ottawa. Ses travaux de recherches portent sur la théorie queer, la mémoire collective et l'affect. Dans le passé, il a œuvré de concert avec des organismes de défense des droits LGBT, des centres de prévention du SIDA et des agences dans le domaine de la déficience intellectuelle. Enfin, il s'est joint récemment au Groupe de recherche sur l'esthétique et la politique de l'image à titre de membre auxiliaire. Son parcours académique a été gracieusement financé par le Conseil de recherche en sciences humaines du Canada. 